\title{
Parameters identification for combined forming of complex shaped sheet metal components
}

\author{
Pashkov Andrey E. \\ Institute of aircraft and machine building and transport \\ (IAMBT) \\ Irkutsk National Research Technical University \\ Irkutsk, Russia \\ pashkov@istu.edu \\ Malashchenko Aleksander.Y. \\ Institute of aircraft and machine building and transport \\ (IAMBT) \\ Irkutsk National Research Technical University \\ Irkutsk, Russia \\ mlk17@mail.ru
}

\begin{abstract}
The article analyzes methods used to identify technological parameters for combined forming of double curved skins. The combined method involves elastic plastic bending and shot peening. The results of finiteelement simulation of these technological processes and their combinations are described. The results of experimental verification of the methods are presented.
\end{abstract}

Keywords—skin, shot peening, bending, finite-element simulation, double curved components.

\section{INTRODUCTION}

For manufacturing large panel- and skin-type parts applied in aircraft and shipbuilding industries, Irkutsk National Research Technical University developed a method combining elastic plastic bending and shot peening (SP) [9]. The key technological advantage of the method is division of the process into simple controllable stages which ensures stability, accuracy and rapid adaptation for manufacturing parts with different designs. At the first stage, the lengthwise curvature is formed with regard to its changes after the SP process. At the second stage, shot peening is used on the aerodynamic surface to create a theoretical shape of the part. Surface roughness formation during shot peen forming is provided by grinding operation [6,7]. Fig. 1 shows the scheme of a theoretical contour of the upper wing panel with a distribution of longitudinal and lateral curvature radii.

\author{
Pashkov Aleksander A. \\ Institute of aircraft and machine building and transport \\ (IAMBT) \\ Irkutsk National Research Technical University \\ Irkutsk, Russia \\ pashk0ff@mail.ru
}

Zarak TatiyanaV.

Institute of aircraft and machine building and transport (IAMBT)

Irkutsk National Research Technical University

Irkutsk, Russia

tv_zar@istu.edu

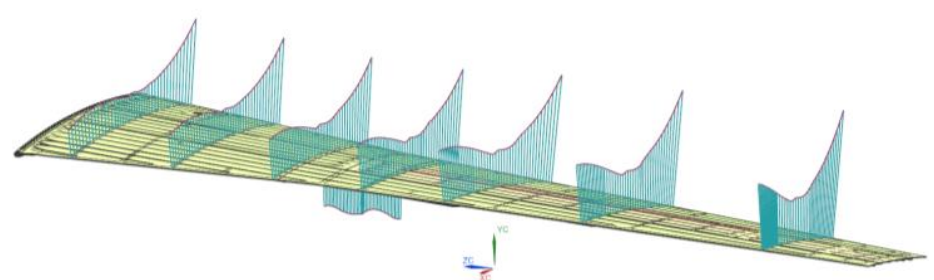

Fig. 1. Distribution of radii of longitudinal and lateral aerodynamic surface curvature for standard wing panels

Increasingly stringent requirements for aircraft products show the need for using CNC machines [10]. As a part of the projects aiming at developing high-productivity production in Irkutsk Aviation Plant (IAP) supported by the Ministry of Education and Science of Russia, CNC-based skin forming machines, including a three-rolled sheet bending machine I2222BM Series, a rolling machine for local plastic deformation of stiffened ribs, a shot peening and finishing machine UDF-4, were developed (see Fig. 2).

For efficient practical application of these machines, special software was developed. It is used for calculating SP parameters based on CAD-models of parts and automated preparation of control software for UDF-4 [3]. In this research, SP parameters are determined for checkpoints of part sections based on the required bending torque by formula:

$$
M_{x}=0,09375 k_{n} k_{o} E H_{y}^{3} \kappa_{x 0},
$$

where $k_{n}$ - factor accounting a decrease in the required curvature under rough forming, $k_{n}=0,7-0,9 ; k_{o}$ - factor accounting the effects of previously machined sections; $H_{y}$ gage section thickness for a workpiece equal to the real one in the moment of inertia and characterizing the bending 
resistance in a relevant direction; $\kappa_{x 0}$ - required lateral curvature of the neutral section layer.

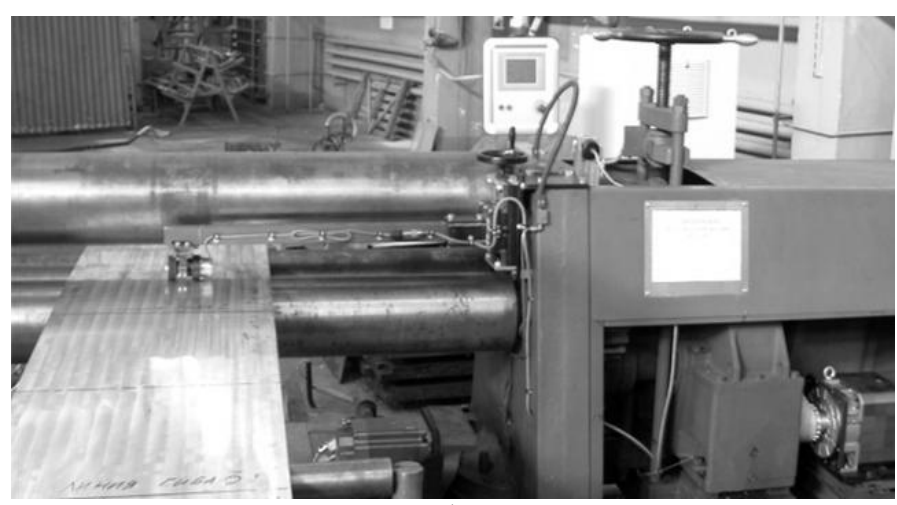

a)

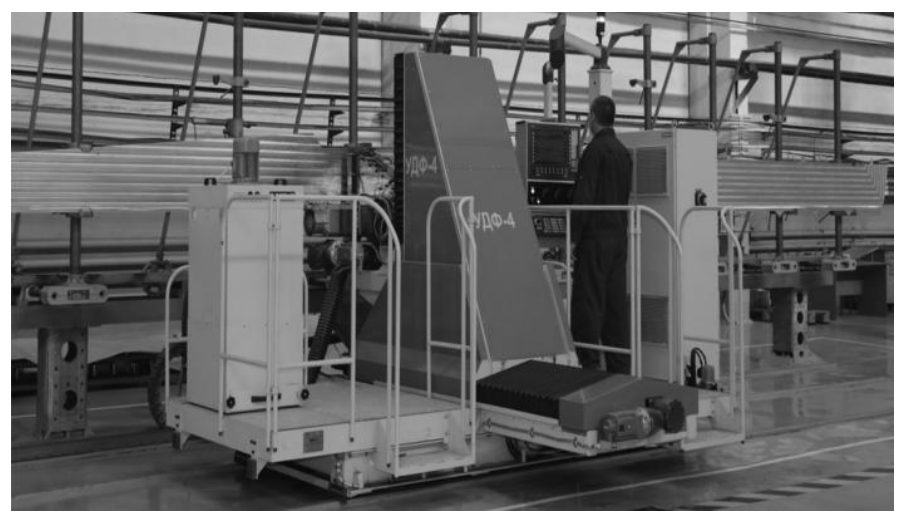

b)

Fig. 2. Machines for combined skin forming: a) I2222BM Series sheet bending machine; б) UDF-4 shot peening machine

\section{COMMON PROBLEMS OF EXISTING TECHNOLOGY}

In formula (1), the values of section thickness and curvature are calculated using a CAD-based computer model. Practical approbation of the method showed that it delivers positive results when calculating forming parameters for line sections. For double-curved sections, calculation errors for the required bending torque increase. Combination of bending and SP process for double-curved sheet metal components is a complex technological task. First, it is necessary to determine bending parameters with regard to changes in shapes. Second, when machining double-curved sections, varying workpiece rigidity influences the shape. Third, effects of the initial state of stress creating at the bending stage need to be identified.

Shape variation for a bended workpiece during the SP process can be described by formulas:

$$
\begin{aligned}
\kappa_{y}^{p f} & =\kappa_{y}^{b}-\frac{P_{p f y}}{K E I_{x}}\left(Z_{0 y}-z_{c y}^{p f}\right) ; \\
\kappa_{x}^{p f} & =\frac{P_{p f x}}{K E I_{y}}\left(Z_{0 x}-z_{c x}^{p f}\right)
\end{aligned}
$$

where $\kappa_{y}^{p f}, \kappa_{x}^{p f}-$ longitudinal and lateral curvature components forming in SP $(\kappa=1 / R$, where $R$ - radius of curvature); $\quad \kappa_{y}^{b}-$ longitudinal curvature of the bended workpiece; $P_{p f x}, P_{p f y}$ - components of the specific internal force caused by SP; $Z_{0 x}, Z_{0 y}-$ longitudinal and lateral coordinates of the neutral layer; $z_{c x}^{p f}, z_{c y}^{p f}$-distance from the shot peened surface to the internal force point; $I_{x}, I_{y}-$ second moments of inertia about relevant axes; $E$ - E-modulus; $K-$ strain-stress state ratio. Under two-axis elastic bending $K=$ 1,5. Internal force of the SP process (specific force $P_{p f}$ ) and a force point coordinate are connected with SP modes. Methods for identifying force factors are described in [1]. By their effects on the workpiece shape in "Bending - SP", variables in formula (1) can be divided into three groups:

1) Geometry parameters $I_{x}, I_{y} ; Z_{0 x}, Z_{0 y}$ depending on structural features, fastening methods and workpiece shapes varying in forming.

2) Longitudinal curvature $\kappa_{y}^{b}$ formed during the bending process which aims to produce required workpiece curvature and compensate for longitudinal curvature formed in the SP process. Longitudinal curvature $\kappa_{y}^{b}$ influences:

- the moment of inertia $I_{x}$ determining lateral workpiece rigidity;

- the residual stresses formed in the bending process. They influence the strain-stress state and internal force factors of the SP process.

3) Internal force factors of the SP process $P_{p f}, z_{c}$ depending on the technological parameters (diameters, ball speed, machining time) and the initial strain-and-stress state determined by residual and fixturing stresses.

\section{DEVELOPMENT OF THE METHOD FOR IDENTIFYING BENDING-SP PARAMETERS}

Analysis of SP parameters by formulas (2) is challenging due to a number of interdependent factors. The methods described in [2] are based on assumptions impairing calculation accuracy. When forming skins with such structural elements as pockets, ribs, thickened parts etc., supporting sections are subjected to plastic strains while sheet is subjected 
to elastic ones and enhances a spring effect after unloading (see Fig. 3). Therefore, bending parameters calculation methods applied to sheet metal components [4] are not accurate for setting parameters of the sheet bending machine without conducting experimental works. As described in [8], the finite element method is the most accurate one for identifying elastoplastic strain parameters. The method for identifying bending-SP parameters for double-curved components is described below.

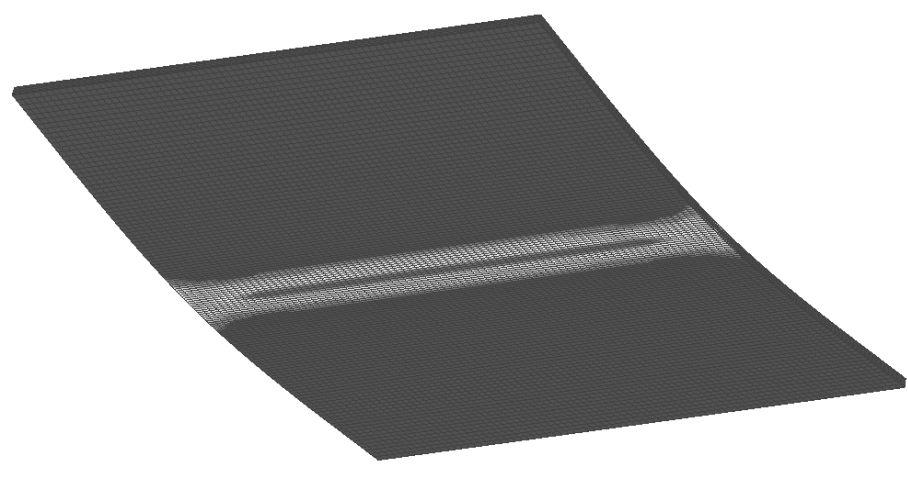

a)

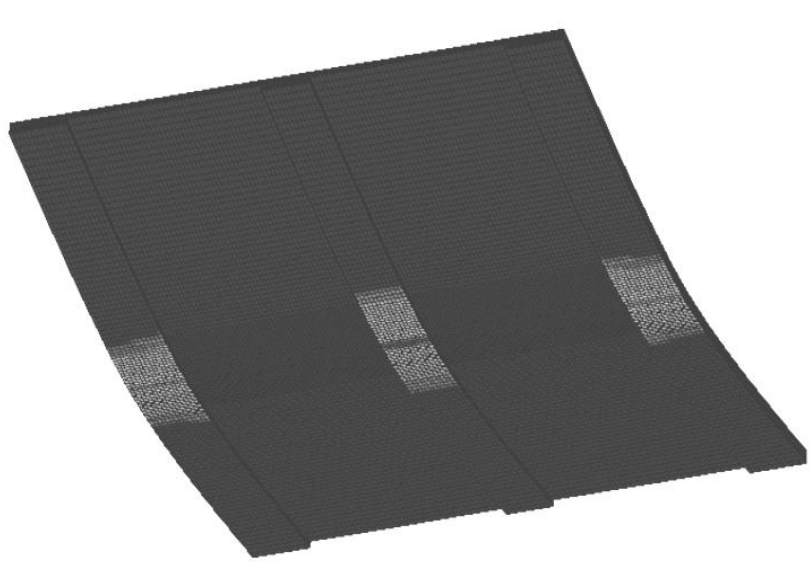

b)

Fig. 3. Distribution of plastic deformations when bending sheet (a) and a skin section (b) at the same thickness of the cross-section area

Finite-elements are calculated in LSTC LS-Dyna. As a simulation object, a CAD model of the initial flat workpiece with dimensions corresponding to the double curved area is used. Using a CAE-system pre-processor, let us simplify the workpiece geometry by eliminating roundness, fillets, bevels and other elements and divide the workpiece into rectangular segments.

It is difficult to simulate the SP process when shots are impacting with the real workpiece surface due to high labor intensity of calculation model development and long duration of calculations. Therefore, to simulate the SP process, let us load a workpiece with extension forces (see Fig. 4) exerted on grid nodes located at $z_{c}$. The simulation result is shown in Fig. 5. The model helps determine the distribution of longitudinal and lateral workpiece curvatures $\kappa_{j}^{p f}(j=x, y)$ and values of required internal forces.

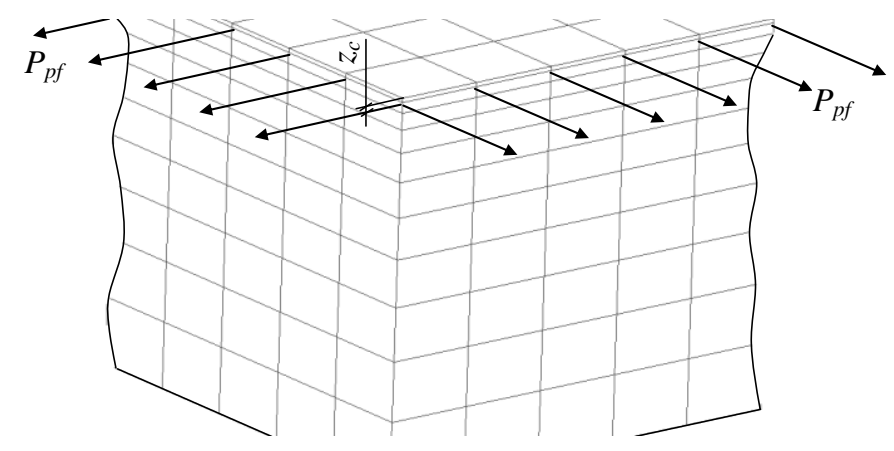

Fig. 4. The scheme of extension force application to grid nodes in finiteelement simulation of the SP process

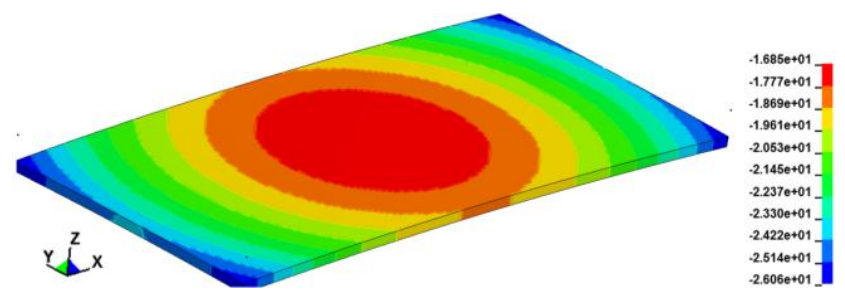

Fig. 5. Movement of nodes of the finite-element model along the OZ axis

Bending parameters can be calculated by formula:

$$
\kappa_{y}^{b}=\kappa_{y}-\kappa_{y}^{p f}
$$

where $\kappa_{y}^{b}-$ longitudinal curvature resulted from elastoplastic bending ( $\kappa=1 / R, R$-radius of curvature); $\kappa_{y}-$ required value of the longitudinal workpiece section curvature; $\kappa_{y}^{p f}$ - longitudinal curvature resulted from SP aiming at forming the required lateral curvature $\kappa_{x}$. As laws of signs in (2), let us assume that the direction of lateral workpiece curvature is positive.

The bending process is simulated to determine the setting parameters for the sheet bending machine required for forming the curvature $\kappa_{y}^{b}$ (see Fig. 6). 


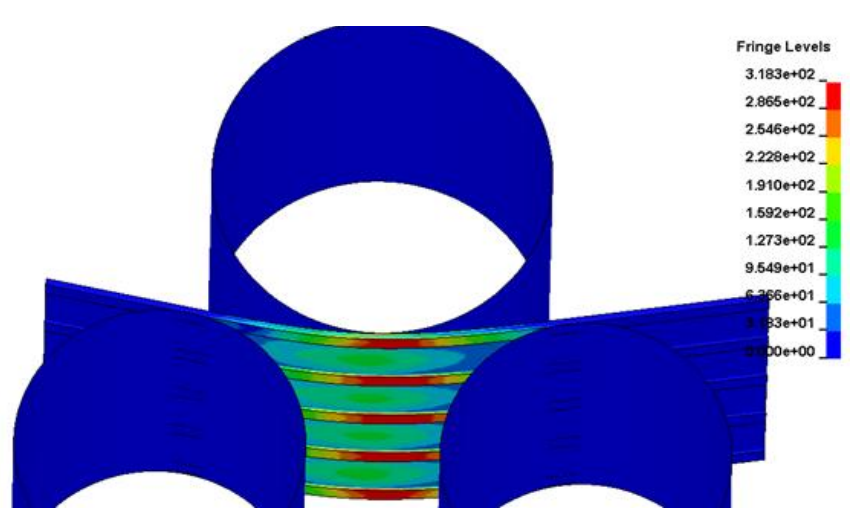

a)

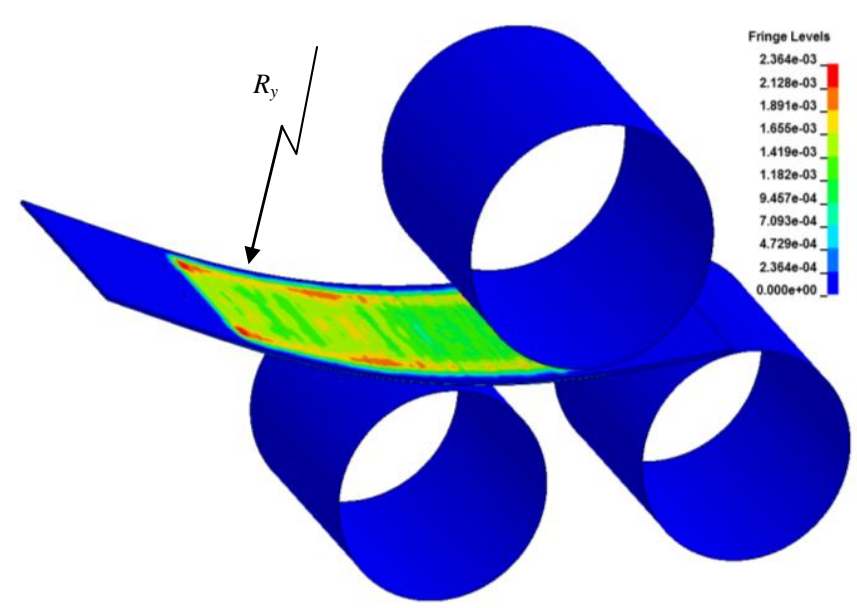

b)

Fig. 6. Distribution of Mises stresses (a) and effective plastic strains (b) in the sheet sample after bending-rolling

For simulation, the explicit method was used as far as a small integration step is beneficial for solving non-linear material behavior and geometry tasks (contact interactions and friction).

Interaction of rolls and the workpiece is set with a surface to surface contact implemented in LS-DYNA. The interaction moment is determined automatically when the surface of one body contacts the surface of the other one. The friction ratio is calculated by formula [5]:

$$
\mu_{c}=F D+(F S+F D) e^{-D C V_{r e l}}
$$

where $F D=0.45-$ dynamic friction factor, $=0.60 F S-$ static friction factor, $D C$ - exponential extinction factor, $V_{r e s}-$ relative speed of contacting surfaces.

Anisotropy of mechanical material properties requires plastic strain resistance testing when extending in the principal anisotropy axes and shifting to the principal axes. When bending wide workpieces, flat strain-stress states occur, therefore when calculating setting skin bending parameters, a simplified model of elastoplastic material is used. It takes into account only longitudinal and lateral differences of mechanical properties. To determine mechanical material properties, let us test the samples made from workpiece material for longitudinal and lateral extension.

The anisotropic elastoplastic material model is used. The model is based on von Mises yield criterion [5]:

$$
\begin{gathered}
2 f\left(\sigma_{i j}\right)=F\left(\sigma_{y}-\sigma_{z}\right)+G\left(\sigma_{z}-\sigma_{x}\right)+H\left(\sigma_{x}-\sigma_{y}\right)+2 \mathrm{~L} \tau_{y z}^{2}+ \\
+2 M \tau_{z x}^{2}+2 N \tau_{x y}^{2}=1
\end{gathered}
$$

where $F, G, H, L, M, N$ - anisotropy factors.

Due to the application of rolled sheet, the orthotropic model was used. To calculate anisotropy factors, the material was tested for longitudinal and lateral axial extension.

The relationship between strains and stresses was linear ( $\sigma=E \varepsilon)$. For the plastic zone, the relationship was in the form of a loading curve approximated by a set of straight sections.

To eliminate vibrations in the areas of contact of the workpiece and the bending rolls, two damping models were applied: the Rayleigh damping model and the global damping model in deformable body units and in body centers of mass. Damping acceleration was proportional to the body mass. It was calculated by formula [5]:

$$
a^{n}=M^{-1}\left(P^{n}-F^{n}-F_{\text {damp }}^{n}\right)
$$

where $M$ - lumped mass matrix, $P^{n}$ - external load vector, $F^{n}$ - internal force vector, $F_{\text {damp }}^{n}-$ damping force.

Due to elastic nature of strains, the calculation of a spring rate is a linear task. Non-linear conditions were neglected by eliminating finite-element roll grids when exporting damping workpieces. To save calculation resources, elastic restitution was calculated using an implicit method which helps form the stress-strain state.

These simulation methods can be used to determine setting sheet bending machine parameters for achieving the required longitudinal curvature and the residual stress state of the bended and fixtured workpiece.

Let us simulate the SP process with regard to the stressstrain state of the bended workpiece.

To assess fixturing effects, let us use a workpiece model with longitudinal residual curvature $\kappa_{y}^{b}$ which will be loaded with proportionally increasing forces $P_{p f}$. To decrease negative effects of high rigidity in the double curved area, the 
following fixturing methods can be used: longitudinal fixturing; complete rectification; creation of the preliminary contour of positive curvature. Fixturing preceded by preliminary elastic bending increases SP performance due to decreasing cross section rigidity in the double curved area, decreasing required bending moment and reduced machining time. Let us simulate the fixturing process using spherical supports positioned on both sides of the longitudinal workpiece symmetry line (see Fig. 7). The supports are absolutely rigid bodies. Under internal forces, the workpiece bends only in X0Z.

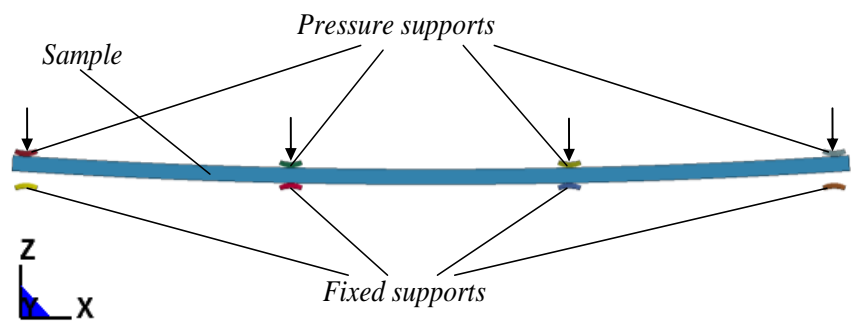

Fig. 7. Longitudinal part fixturing when simulating the SP process

Fig. 8 shows calculation results presented as distributions of grid nodes movements for the finite element model

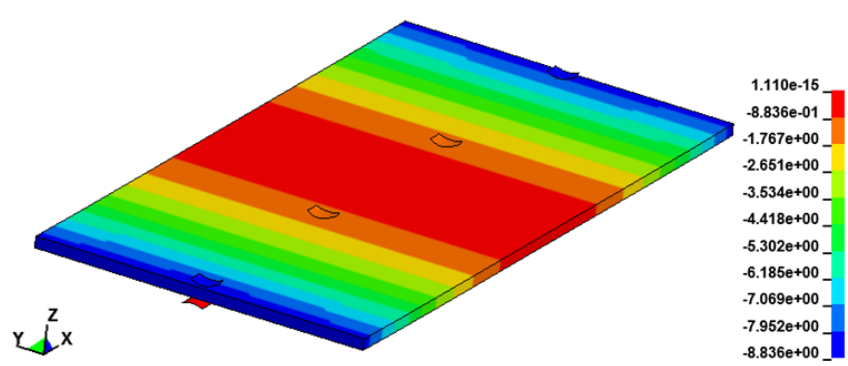

a)

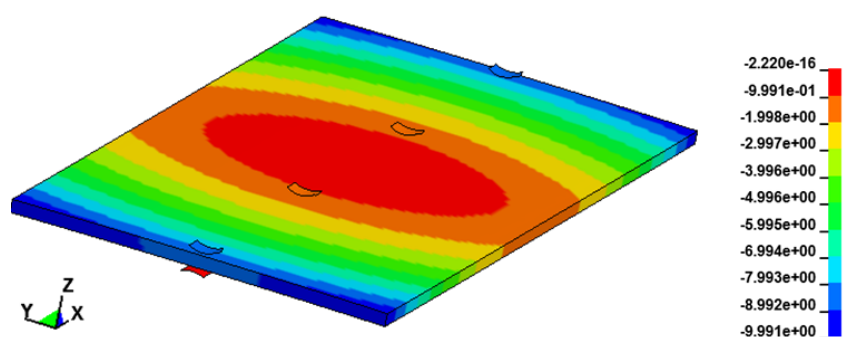

b)

Fig. 8. Distribution of movements along Z-axis in the sample (V95pchT2, $\left.\mathrm{H}=10 \mathrm{~mm}, \quad \boldsymbol{R}_{y}=-5 \mathrm{~m}\right)$ : a) resulted from rectification $\left(\boldsymbol{R}_{y}^{f i x}=\infty\right)$ and longitudinal fixturing; $b$ ) resulted from SP

Fixturing effects can be determined by $K_{1}$

$$
K_{1}=P_{p f}^{f i x} / P_{p f 1}
$$

where $P_{p f}^{f i x}, P_{p f 1}-$ specific internal forces needed for producing required lateral curvature $\kappa_{x}$ of the bended part which is elastically deformed by fixturing / in a free flat state. Using the simulation results, let us calculate a radius of curvature $\boldsymbol{R}_{y}^{f i x}$ which is optimal in terms of process intensification.

To determine the effect of the initial strain-and stress state resulted from longitudinal bending and fixturing, can be used simulation of the implementation of a $3,5 \mathrm{~mm}$ ball into the plate $100 \times 20 \times 10 \mathrm{~mm}$ (length $\times$ width $\times$ thickness $)$ in size subjected to elastoplastic bending and rectification. To this end, after workpiece bending and fixturing have been completed, the deformed model grid is imported into a new calculation file. To eliminate move-functions, let us limit the degree of freedom of model nodes along all edges except for the machined surface. Fig. 10 shows the simulation results. The ball is implemented into the plastically deformed model area shown in Fig. 9.

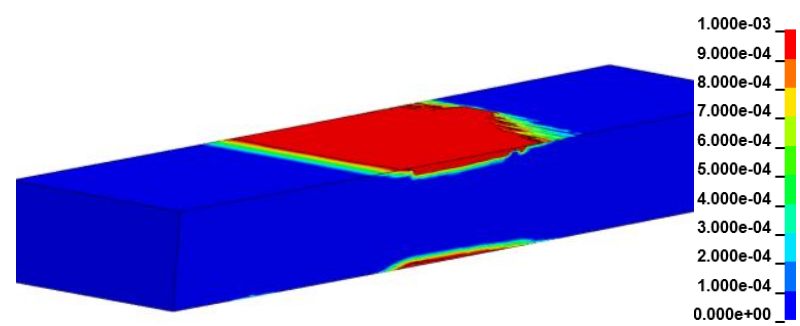

Fig. 9. Distribution of residual strains in the sample model during the elastoplastic bending process at Rres $=-5 \mathrm{~m}$

Fig. 10 shows distribution of initials stresses in the surface sample layer after ball machining.

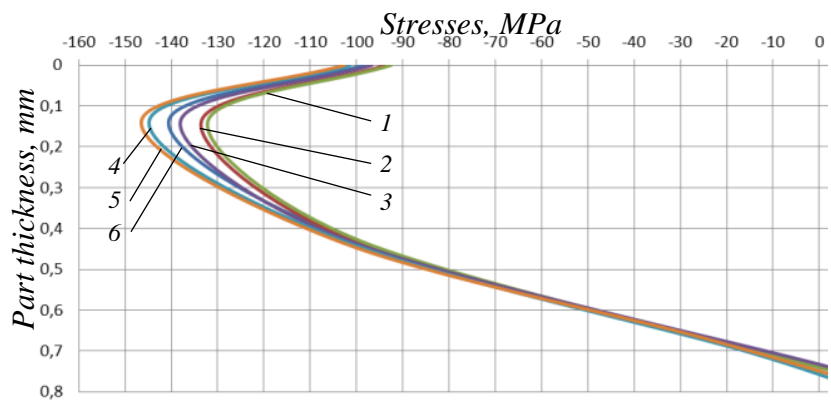

Fig. 10. Distribution of normal stresses after ball implementation into the surface sample layer $4 \mathrm{~mm}$ in thickness: 1 - residual bending stresses $\mathrm{R}_{\mathrm{res}}=-5$ $\mathrm{m} ; 2$ - elastic bending stresses $\mathrm{R}=-10 \mathrm{~m} ; 3$ - bending stresses after elastic rectification to the $2 \mathrm{D}$ state; 4 - elastic bending stresses $\mathrm{R}=10 \mathrm{~m} ; 5$ - elastic bending stresses $\mathrm{R}=5 \mathrm{~m} ; 6$ - unstrained sample. 
Assuming the similar geometry of the diagrams, let us calculate $K_{2}$ which accounts the residual stress state of the bended and fixtured part:

$$
K_{2}=\sigma_{p f}^{s s} / \sigma_{p f 1}
$$

where $\sigma_{p f}^{s s}, \sigma_{p f 1}-$ maximum values of residual stresses calculated using the diagrams built with / without regard to the initial strain-and-stress state of the part respectively.

Let us simulate the SP process which involves two stages: rough machining and finishing, including part shape measurement.

Rough machining of the workpiece fixtured with a radius of longitudinal curvature $\boldsymbol{R}_{y}^{f i x}$ is simulated with proportionally increasing forces $P_{p f 2}$ applied to workpiece sections.

$$
P_{p f 2}=K_{1} K_{2} K_{3} P_{p f 1}
$$

where $K_{3}-$ factor determining the accordance of the lateral curvature with the required curvature $\kappa_{x}, K_{2}=$ $0,7 \ldots 0,9$. Lateral curvature is determined after the preliminary SP stage has been completed.

When simulating the final SP stage (Fig. 7), supports fixturing the workpiece have to be moved so that to ensure compliance of the longitudinal curvature with the theoretical contour of the part. The required lateral curvature $\kappa_{x}$ is achieved by iteration changes of force $P_{p f}$. Then, values of the required specific internal force $P_{p f 3}$ and machining time $t_{p f 3}$ are calculated.

Using cross-section deflection measurement results, let us calculate the required adjusted value of force $P_{p f 3}^{r}$ with regard to the linear relationship between the curvature and the specific internal force:

$$
P_{p f 3}^{r}=P_{p f 3} \frac{f_{x 2}^{m}}{f_{x 2}}
$$

where $f_{x 2}, f_{x 2}^{m}$ - calculated and measured deflection values in checkpoints.

By the required force values, using the method described in [1], let us calculate corrected workpiece machining time $t_{p f 3}^{r}$ and machining time $t_{p f 4}$ at the final SP stage.

$$
t_{p f 4}=t_{p f 3}^{r}-t_{p f 2} .
$$

Thus, to develop finishing SP control software, feed speed for gage sections without changing wheel blasting rotation frequency has to be calculated accounting $t_{p f 4}$.

\section{CONCLUSIONS}

To test the methods for determining SP conditions, structurally similar samples (SSS) of the civil aircraft wing skin sections made from V95 alloy metal were used (see Fig. 11).

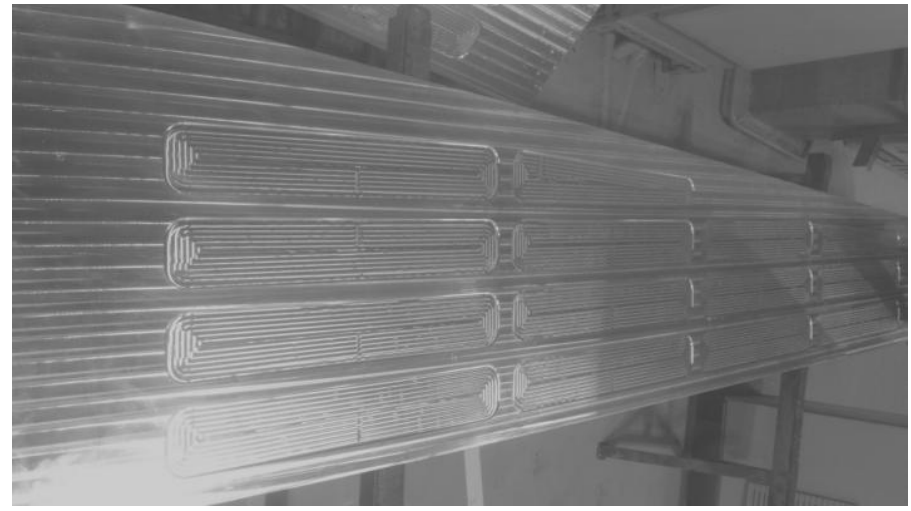

a)

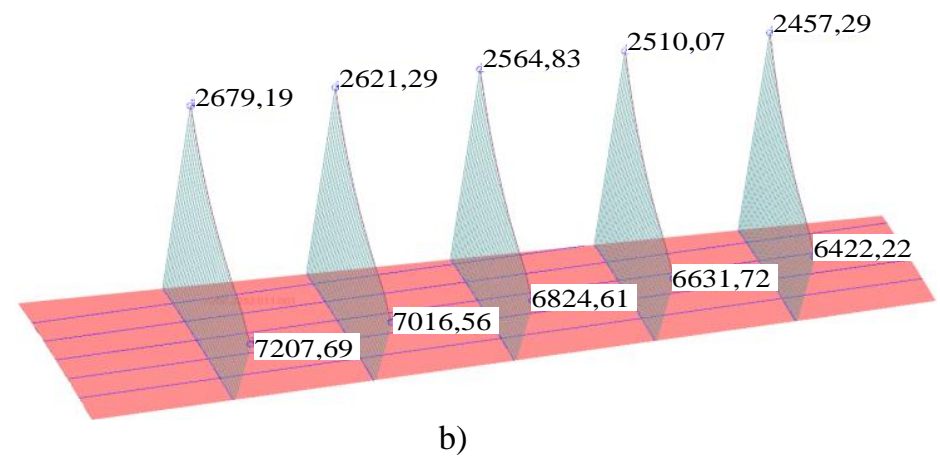

Fig. 11. A general view of the SSS of the civil aircraft wing skin section (a) and distribution of radii of lateral curvature of the theoretical contour (b)

SSSs were bended and rolled using a sheet bending machine based on recommendations in [Ошибка! Источник ссылки не найден., Ошибка! Источник ссылки не найден.]. To determine a residual radius of the longitudinal curvature, longitudinal deflection was measured in checkpoints using an indicator plate with a gauge length of $200 \mathrm{~mm}$ equipped with a clock-type head ICh-10 reading in $0,01 \mathrm{~mm}$.

When shot peening, the SSSs were fixtured in the UDF-4 fixturing system as shown in Fig. 12. Three fixturing methods 
were applied: longitudinal fixturing $R_{y}^{f i x}=-10 \mathrm{~m}$, complete rectification, creation of the preliminary contour $R_{y}^{f i x}=10 \mathrm{~m}$.

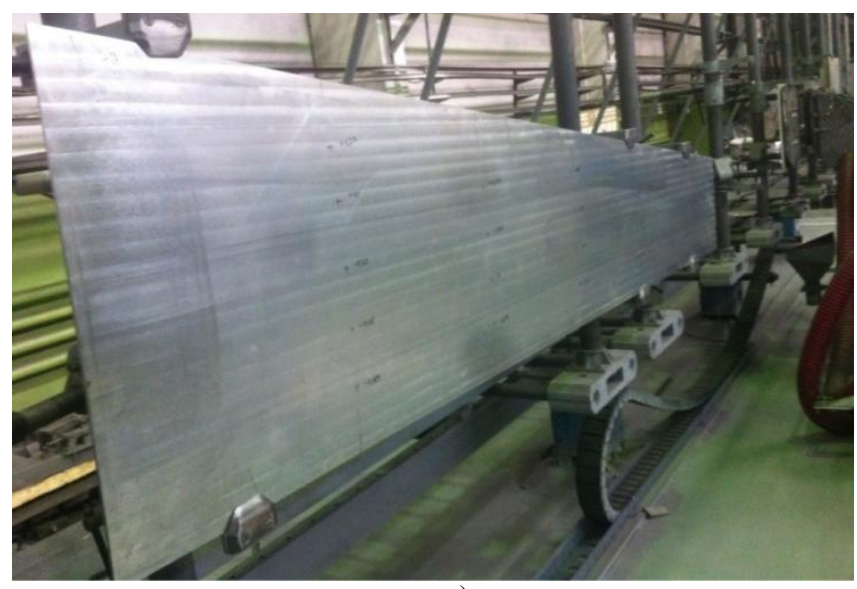

a)

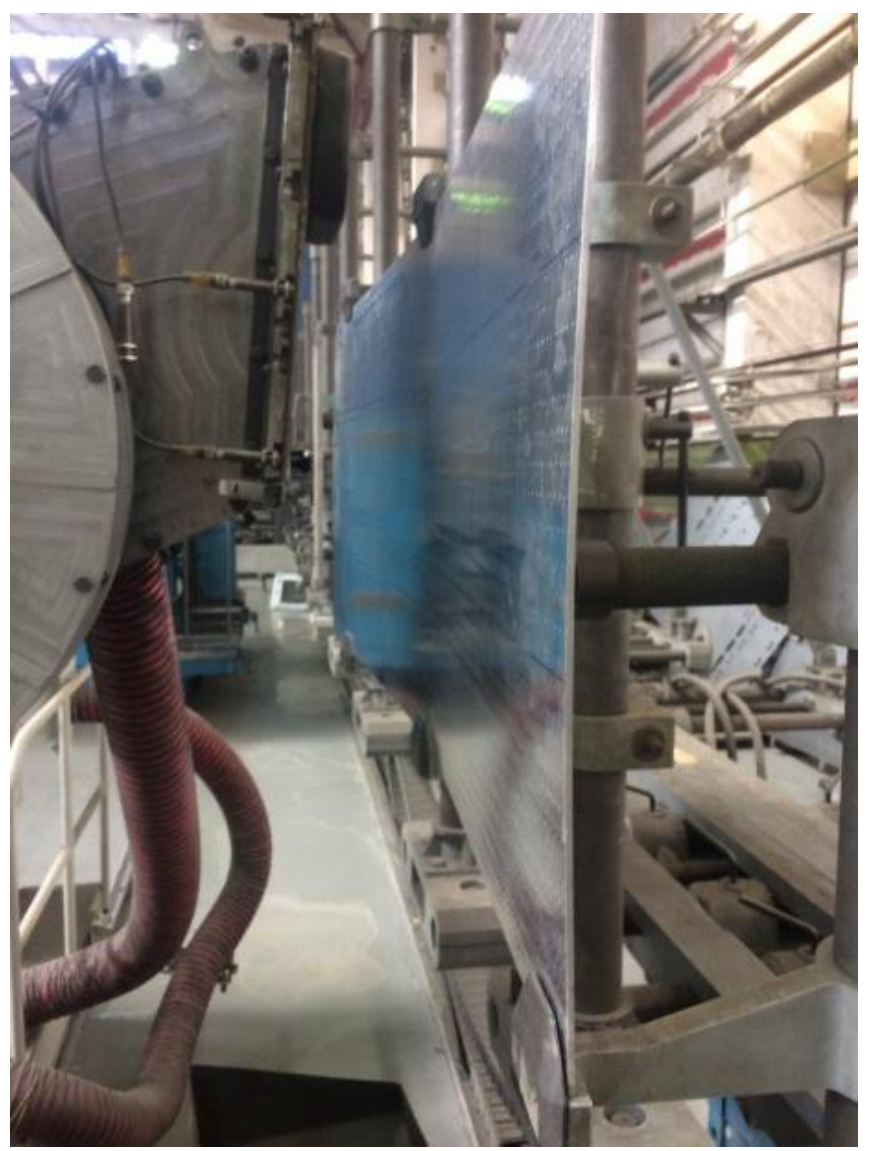

b)

Fig. 12. SSS fixturing (a) in UDF-4 when shot peening (b)

After the preliminary SP stage has been completed, the SSSs were released, and lateral deflection was measured in the checkpoints. After finishing, lateral contour control was carried out along rib axes using cross-section contour patterns (CSCP). Contour deviations were measured by probes.
Measurements showed that the combined method helped achieve the required surface shape. Lateral contour deviations along rib axes measured with CSCP were within the normal range of 0,4 to $0,8 \mathrm{~mm}$ without loading.

\section{References}

[1] Y.S. Andryashina, "Automated calculation of shot peening parameters for large panels", App. the journal of the samara scientific center of the russian academy of sciences, pp. 305 - 308, December 2013 [5th AllRussian Conference "Modern advanced innovation technology", p. 550 , 2013]

[2] Diyak A.Y., The system of automated sheet metal forming control program development using contact CNC-based shot-peening machines author's abstract. Irkutsk, 2005. p. 3 - 19.

[3] Pashkov A.E. "Software calculation module for panel shot peening parameters", Author's certificate of the Russian Federation, A2014611955, December, 2013.

[4] G.B. Aguiar, G.M. Barbosa, G.F. Batalha, "Sheet bending theory applied to a three roll process", Brazil, 2015, pp $1-8$.

[5] J.O. Hallquist, LS-DYNA Keyword User's Manual, version 970, Vol. 2, Augest, 2016, pp 521 - 523

[6] V P Koltsov, Le Tri Vinh, D A Starodubtseva "Surface roughness formation during shot peen forming", IOP Conference Series: Materials Science and Engineering, Vol. 327, Tomsk, Russia, December 2018, pp $1-8$, [Processing Equipment, Mechanical Engineering Processes and Metals Treatment (The XI International Conference on Mechanical Engineering, Automation and Control Systems, 2017.)

[7] V. Koltsov, D. Starodubtseva "Investigation of Traces of Interaction between Flap Wheel and Aluminum Alloy Plain Surface", Procedia Engineering, Vol. 206, 2017 pp. 473-478.

[8] A.Y Malashchenko, "Research of technological capacities of the process of long-length skin forming by combination of roll-bending and peen forming processing”, Russian Aeronautics, Vol. 59, № 2, 2016, pp. 254-258.

[9] A.E. Pashkov, A.A. Pashkov, V.P. Koltsov, "Complex method of peen forming and shot peening of aircraft structural components" Proceedings of the international conference «Actual issues of mechanical engineering», Vol. 133, 2017, pp. 585-591.

[10] J.A. Vaccari, "Peen forming enters computer age American Machinist", June, 1985, pp. 91-94. 